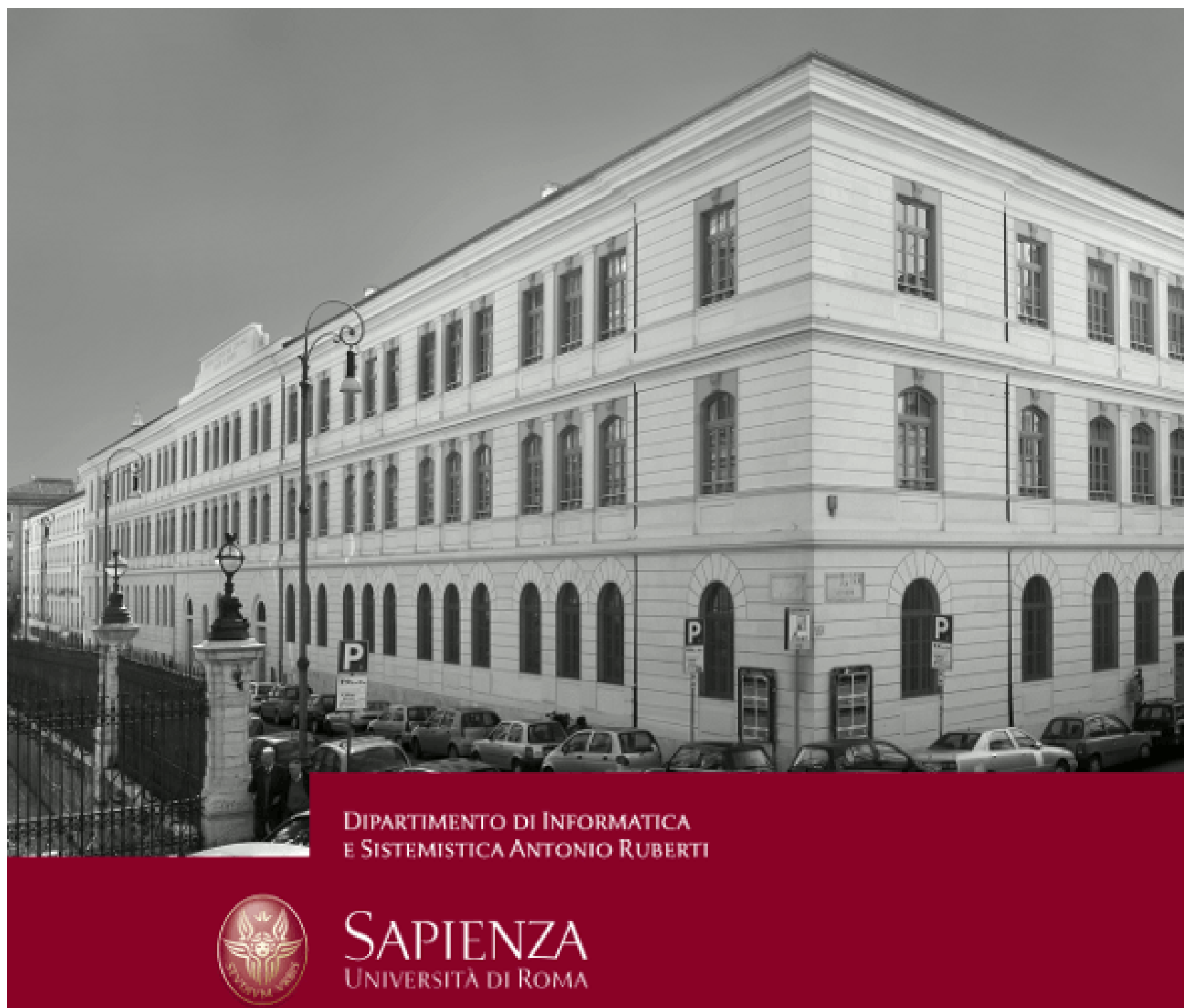

Unconstrained formulation of standard quadratic optimization problems

Immanuel M. Bomze

Luigi Grippo

Laura Palagi

Technical Report n. 12, 2010 


\title{
Unconstrained formulation of standard quadratic optimization problems
}

\author{
Immanuel M. Bomze* Luigi Grippo ${ }^{\dagger} \quad$ Laura Palagi $^{\dagger}$
}

This version: September 22, 2010

Tech. Report DIS-La Sapienza Vol.2(2010), No. 12

\begin{abstract}
A standard quadratic optimization problem (StQP) consists of finding the largest or smallest value of a (possibly indefinite) quadratic form over the standard simplex which is the intersection of a hyperplane with the positive orthant. This NP-hard problem has several immediate real-world applications like the Maximum-Clique Problem, and it also occurs in a natural way as a subproblem in quadratic programming with linear constraints. We propose unconstrained reformulations of StQPs, by using different approaches. We test our method on clique problems from the DIMACS challenge.
\end{abstract}

Key Words: standard quadratic optimization, maximum clique, exact penalization.

\section{Introduction and preliminaries}

\subsection{Standard quadratic optimization problems and related problems}

In this paper we consider the standard quadratic optimization problem (StQP) of the form

$$
\min \left\{\varphi(\mathbf{y})=\frac{1}{2} \mathbf{y}^{\top} A \mathbf{y}: \mathbf{y} \in \Delta\right\}
$$

where $\Delta$ denotes the standard simplex in $n$-dimensional Euclidean space $\mathbb{R}^{n}$, namely

$$
\Delta=\left\{\mathbf{y} \in \mathbb{R}_{+}^{n}: \mathbf{e}^{\top} \mathbf{y}=1\right\}
$$

and $A=\left[a_{i j}\right] \in \mathbb{R}^{n \times n}$ is a symmetric $n \times n$ matrix; $\mathbf{e}$ is the $n$-vector of all ones and $\mathbf{y}^{\top}$ denotes the transposed vector while $I$ denotes the $n \times n$ identity matrix. In the sequel, $\|\mathbf{x}\|=\sqrt{\mathbf{x}^{\top} \mathbf{x}}$ always denotes the Euclidean norm of a vector $\mathbf{x} \in \mathbb{R}^{n}$.

StQP is an NP-hard problem that has arise many immediate applications, among them a continuous formulation of the Maximum-Clique Problem. For more details about StQP, we refer to [3] and [4].

\footnotetext{
*Dept. of Statistics and Decision Support Systems, University of Vienna, Austria

${ }^{\dagger}$ Università di Roma La Sapienza - Dipartimento di Informatica e Sistemistica A. Ruberti, Italy.
} 
In [6] a quartic formulation of the StQP has been proposed, which uses the substitution $y_{i}=x_{i}^{2}$, to get rid of the sign constraints $y_{i} \geq 0$. Then the condition $\mathbf{e}^{\top} \mathbf{y}=1$ reads $\|\mathbf{x}\|^{2}=1$, and we get the following ball constrained problem (BQP)

$$
\min \left\{\Phi(\mathbf{x})=\frac{1}{2} \mathbf{x}^{\top} X A X \mathbf{x}:\|\mathbf{x}\|^{2}=1\right\}
$$

where we denote by $X$ the diagonal matrix with elements $x_{i}$.

The two constrained problems (1) and (2) are not fully equivalent in the sense that spurious points satisfying necessary optimality conditions can be created in passing from Problem (1) to Problem (2). However, by using second-order conditions, we are able to identify such spurious points.

Using the special structure of the constraint in (2), in [6] an simple unconstrained formulation (UQP) of (2) has been proposed that in turn can be used to find a local solution of the StQP (1). Thus problem (2) can be solved by means of a single unconstrained minimization as follows:

$$
\min \left\{P_{\varepsilon}(\mathbf{x})=\Phi(\mathbf{x})\left(3-2\|\mathbf{x}\|^{2}\right)+\frac{1}{\varepsilon}\left(\|\mathbf{x}\|^{2}-1\right)^{2}: \mathbf{x} \in \mathbb{R}^{n}\right\},
$$

where $\varepsilon$ can be freely choosen within $(0, \bar{\varepsilon}]$, and the upper bound $\bar{\varepsilon}$ is easily calculated (see [6] for details).

The definition of an unconstrained formulation allows to use very efficient methods for the solution able to tackle very large scale problems. In this paper we further exploit the possibilities of defining two alternative unconstrained formulations of the StQP (a) using the quartic formulation (2) and (b) revising a formulation proposed in [3].

The relations of the solution of the StQP with those of the two different unconstrained formulations are studied. A numerical study of the performance on the DIMACS data set of the maximum clique problems is performed.

After a review of notations, optimality conditions for StQP in Section 1.2, we introduce in Section 2 a new unconstrained formulation based on the ball constrained Problem (2) is proposed. In section 3 a new unconstrained formulation based on the simple formulation of StQP given in [3] has been proposed. In Section 4, numerical results are reported with performance comparison.

\subsection{Optimality conditions for StQP}

Since the constraints are linear, the Karush-Kuhn-Tucker (KKT) conditions are necessary conditions for local optimality and can be stated as follows.

Proposition 1 (KKT necessary condition for StQP problem (KTS)) Let $\overline{\mathbf{y}}$ be a local solution of Problem (1), then scalar $\bar{\lambda}$ exists such that

$$
\left\{\begin{array}{lll}
(A \overline{\mathbf{y}})_{i}+\bar{\lambda}=0 & \text { for } i: & \bar{y}_{i}>0 \\
(A \overline{\mathbf{y}})_{i}+\bar{\lambda} \geq 0 & \text { for } i: & \bar{y}_{i}=0
\end{array}\right.
$$


Further the Lagrange multiplier is uniquely determined by $\overline{\mathbf{y}}$ and we have $\bar{\lambda}=-\overline{\mathbf{y}}^{\top} A \overline{\mathbf{y}}=-2 \varphi(\overline{\mathbf{y}})$

We note that these conditions can be easily re-written as

$$
\begin{aligned}
& \overline{\mathbf{y}}^{\top}(A \overline{\mathbf{y}}+\bar{\lambda})=0 \\
& \overline{\mathbf{y}} \geq \mathbf{o} \\
& A \overline{\mathbf{y}}+\bar{\lambda} \geq \mathbf{o}
\end{aligned}
$$

In the sequel, we will invoke (weak) Second-order necessary optimality conditions for StQP problem (WSS) (cf., e.g., [8], p.61). Note that there are exact second-order characterizations of local optimality which we do not use here since they involve copositivity checks which are NPhard; see, e.g., [1], also for the close relation to characterizations of global optimality in general quadratic optimization problems.

Proposition 2 (Weak second order necessary condition (WSS) for StQP) Let $\overline{\mathbf{y}}$ be a local solution of Problem (1), then in addition to (4) we have that

$$
\mathbf{z}^{\top} A \mathbf{z} \geq 0 \quad \text { for all } \mathbf{z} \in \mathcal{Z}(\overline{\mathbf{y}})=\left\{\mathbf{z} \in \mathbb{R}^{n}: \sum_{i \in \mathcal{I}(\overline{\mathbf{y}})} z_{i}=0, \text { and } z_{i}=0 \text { for all } i \notin \mathcal{I}(\overline{\mathbf{y}})\right\}
$$

where $\mathcal{I}(\overline{\mathbf{y}})$ denotes the "inactive" variables, namely

$$
\mathcal{I}(\overline{\mathbf{y}})=\left\{i: \bar{y}_{i}>0\right\} .
$$

By reordering the vector $\mathbf{z}$ as $\left(\mathbf{z}_{\mathcal{I}}, \mathbf{z}_{\overline{\mathcal{I}}}\right)^{\top}$, and according to this also the matrices, the condition (5) can be rewritten as

$$
\mathbf{z}_{\mathcal{I}}^{\top} A_{\mathcal{I} \mathcal{I}} \mathbf{z}_{\mathcal{I}} \geq 0 \quad \text { for all } \mathbf{z}_{\mathcal{I}} \in \mathbb{R}^{|\mathcal{I}|}: \sum_{i \in \mathcal{I}(\overline{\mathbf{y}})} z_{i}=0
$$

\section{Unconstrained quotient formulation}

\subsection{Optimality conditions}

Starting from the quartic formulation (2) and taking inspiration from the ideas for finding eigenvalues of a matrix, we define a sort of Rayleigh quotient and introduce the following problem

$$
\inf \left\{f(\mathbf{x})=\frac{1}{2} \frac{\mathbf{x}^{\top} X A X \mathbf{x}}{\|\mathbf{x}\|^{4}}: \mathbf{x} \in \mathbb{R}^{n} \backslash\{\mathbf{o}\}\right\}
$$

A similar idea has been used also in relaxation for the max cut problem [7, 10]. Note that Júdice and coworkers consider a different generalized Rayleigh quotient function in their study [12] of the Eigenvalue Complementarity Problem (EiCP). By squaring coordinates to get rid of the 
sign constraints as explained above before (2), the EiCP with positive-definite $n \times n$ matrix $B$ could be rephrased as the related but constrained problem

$$
\min \left\{\frac{\mathbf{x}^{\top} X A X \mathbf{x}}{\mathbf{x}^{\top} X B X \mathbf{x}}:\|\mathbf{x}\|=1, \mathbf{x} \in \mathbb{R}^{n}\right\} .
$$

Some of the following results may apply also to this problem.

Let us consider the transformation $\overline{\mathbf{y}}=T_{R}(\overline{\mathbf{x}})$ with $\bar{y}_{i}=\frac{\bar{x}_{i}^{2}}{\|\overline{\mathbf{x}}\|^{2}}$. The same type of consideration made for the transformation $T$ hold. Hence without loss of generality we can assume in the following that $\mathbf{x} \geq \mathbf{o}$. We denote by $\mathbf{x}=T_{R}^{-1}(\mathbf{y})$ the (partial) inverse transformation, namely $\bar{x}_{i}=+\sqrt{\bar{y}_{i}}$.

Since the function $f$ is constant along rays from the origin (and discontinuous there, unless it is overall constant), we may safely restrict our attention to a domain $D$ which does not contain a small neighborhood of $\mathbf{o}$. Then $f$ is twice continuously differentiable on $D$ and we have for all $\mathbf{x} \in D:^{1}$

$$
\begin{aligned}
\nabla f(\mathbf{x}) & =2 \frac{X A X \mathbf{x}}{\|\mathbf{x}\|^{4}}-\frac{2 x^{\top} X A X \mathbf{x}}{\|\mathbf{x}\|^{6}} \mathbf{x}=\frac{2}{\|\mathbf{x}\|^{4}}\left(I-\frac{1}{\|\mathbf{x}\|^{2}} \mathbf{x x}^{\top}\right) X A X \mathbf{x} \\
& =2 \frac{X A X \mathbf{x}}{\|\mathbf{x}\|^{4}}-\frac{4 f(\mathbf{x})}{\|\mathbf{x}\|^{2}} \mathbf{x}=\frac{2}{\|\mathbf{x}\|^{4}}\left(X A X-2 f(\mathbf{x})\|\mathbf{x}\|^{2} I\right) \mathbf{x}
\end{aligned}
$$

and

$$
\nabla^{2} f(\mathbf{x})=-\frac{4}{\|\mathbf{x}\|^{2}}\left(\mathbf{x} \nabla f(\mathbf{x})^{\top}+\nabla f(\mathbf{x}) \mathbf{x}^{\top}\right)-\frac{4 f(\mathbf{x})}{\|\mathbf{x}\|^{2}}\left(2 \frac{\mathbf{x} \mathbf{x}^{\top}}{\|\mathbf{x}\|^{2}}+I\right)+\frac{2}{\|\mathbf{x}\|^{4}}(2 X A X+\operatorname{Diag}\{A X \mathbf{x}\})
$$

The first order necessary optimality conditions for a feasible point $\overline{\mathbf{x}}$ to be a local solution of Problem (7) require that $\nabla f(\overline{\mathbf{x}})=\mathbf{o}$, that is:

$$
\frac{2}{\|\mathbf{x}\|^{4}}\left(I-\frac{1}{\|\mathbf{x}\|^{2}} \mathbf{x} \mathbf{x}^{\top}\right) X A X \mathbf{x}=\mathbf{o}
$$

Proposition 3 (First order necessary condition (FNR)) Let $\overline{\mathbf{x}} \neq$ o be a local minimizer for Problem (7). Then for all $i$

$$
\bar{x}_{i}=0 \quad \text { or } \quad(A \bar{X} \overline{\mathbf{x}})_{i}=2 f(\overline{\mathbf{x}})\|\overline{\mathbf{x}}\|^{2} .
$$

Proof. The assertion is immediate from (8).

We denote

$$
\mathcal{I}(\overline{\mathbf{x}})=\left\{i: \bar{x}_{i} \neq 0\right\}=\left\{i: \bar{y}_{i}>0\right\} \text {, }
$$

where the last equation follows easily by the definition of the transformation $T_{R}$ and its inverse.

The second-order necessary optimality conditions for Problem (7) involve the Hessian

$$
-\frac{4}{\|\mathbf{x}\|^{2}}\left(\mathbf{x} \nabla f(\mathbf{x})^{\top}+\nabla f(\mathbf{x}) \mathbf{x}^{\top}\right)-\frac{4 f(\mathbf{x})}{\|\mathbf{x}\|^{2}}\left(2 \frac{\mathbf{x} \mathbf{x}^{\top}}{\|\mathbf{x}\|^{2}}+I\right)+\frac{2}{\|\mathbf{x}\|^{4}}(2 X A X+\operatorname{Diag}\{A X \mathbf{x}\}) .
$$

\footnotetext{
${ }^{1}$ We note that $\nabla\left(\|\mathbf{x}\|^{4}\right)=4\|\mathbf{x}\|^{2} \mathbf{x}$ and $\nabla(X A X \mathbf{x})=\operatorname{Diag}\{A X \mathbf{x}\}+2 X A X$.
} 
Theorem 4 (Second order necessary condition (SNR)) Let $\overline{\mathbf{x}} \neq \mathbf{o}$ be a local minimizer for Problem (7). Then

$$
(A \bar{X} \overline{\mathbf{x}})_{i} \geq 2 f(\overline{\mathbf{x}})\|\overline{\mathbf{x}}\|^{2} \quad \text { with equality for all } i \text { where } \bar{x}_{i} \neq 0
$$

and

$$
\bar{X}_{\mathcal{I}} A_{\mathcal{I I}} \bar{X}_{\mathcal{I}} \succeq f(\overline{\mathbf{x}})\|\overline{\mathbf{x}}\|^{2}\left(2 \frac{\overline{\mathbf{x}}_{\mathcal{I}} \overline{\mathbf{x}}_{\mathcal{I}}^{\top}}{\|\overline{\mathbf{x}}\|^{2}}+I_{\mathcal{I}}\right)-\frac{1}{2} \operatorname{Diag}\{A \bar{X} \overline{\mathbf{x}}\}_{\mathcal{I}}
$$

where vector and matrices have been partitioned according to the definition of inactive variables.

Proof. Let us partition the vector $\overline{\mathbf{x}}$ in $\overline{\mathbf{x}}=\left(\overline{\mathbf{x}}_{0} \overline{\mathbf{x}}_{\mathcal{I}}\right)^{\top}$ where $\overline{\mathbf{x}}_{0}$ denotes the components of $\overline{\mathbf{x}}$ such that $\overline{\mathbf{x}}_{i}=0$, whereas $\left(\overline{\mathbf{x}}_{\mathcal{I}}\right)_{i} \neq 0$. Recalling that $\nabla f(\overline{\mathbf{x}})=\mathbf{o}$, we can write the Hessian $\nabla^{2} f(\overline{\mathbf{x}})$ as follows

$$
\nabla^{2} f(\overline{\mathbf{x}})=-\frac{4 f(\mathbf{x})}{\|\mathbf{x}\|^{4}}\left(2\left(\begin{array}{cc}
0 & 0 \\
0 & \overline{\mathbf{x}}_{\mathcal{I}} \overline{\mathbf{x}}_{\mathcal{I}}^{\top}
\end{array}\right)+\|\mathbf{x}\|^{2} I\right)+\frac{2}{\|\mathbf{x}\|^{4}}\left(\left(\begin{array}{cc}
0 & 0 \\
0 & 2 X_{\mathcal{I}} A_{\mathcal{I}} X_{\mathcal{I}}
\end{array}\right)+\operatorname{Diag}\{A X \mathbf{x}\}\right)
$$

Hence the Hessian is a block diagonal matrix composed of two blocks:

$$
\nabla^{2} f(\overline{\mathbf{x}})=\left(\begin{array}{rl}
\left(\nabla^{2} f(\overline{\mathbf{x}})\right)_{00} & 0 \\
0 & \left(\nabla^{2} f(\overline{\mathbf{x}})\right)_{\mathcal{I I}}
\end{array}\right)
$$

The first one is

$$
\left(\nabla^{2} f(\overline{\mathbf{x}})\right)_{00}=\frac{2}{\|\overline{\mathbf{x}}\|^{4}}\left(-2 f(\overline{\mathbf{x}})\|\overline{\mathbf{x}}\|^{2} I_{0}+\operatorname{Diag}\{A \bar{X} \overline{\mathbf{x}}\}_{0}\right)
$$

which is diagonal in itself. The second one is:

$$
\left(\nabla^{2} f(\overline{\mathbf{x}})\right)_{\mathcal{I} \mathcal{I}}=-\frac{4 f(\mathbf{x})}{\|\mathbf{x}\|^{4}}\left(2 \overline{\mathbf{x}}_{\mathcal{I}} \overline{\mathbf{x}}_{\mathcal{I}}^{\top}+\|\mathbf{x}\|^{2} I_{\mathcal{I}}\right)+\frac{2}{\|\mathbf{x}\|^{4}}\left(2 X_{\mathcal{I}} A_{\mathcal{I} \mathcal{I}} X_{\mathcal{I}}+\operatorname{Diag}\{A X \mathbf{x}\}_{\mathcal{I}}\right) .
$$

The unconstrained second order necessary conditions requires that $\nabla^{2} f(\overline{\mathbf{x}}) \succeq O$, hence we must have:

$$
(A \bar{X} \overline{\mathbf{x}})_{i} \geq 2 f(\overline{\mathbf{x}})\|\overline{\mathbf{x}}\|^{2} \quad \text { for all } i \text { with } \quad \bar{x}_{i}=0
$$

and

$$
2 X_{\mathcal{I}} A_{\mathcal{I} \mathcal{I}} X_{\mathcal{I}}+\operatorname{Diag}\{A X \mathbf{x}\}_{\mathcal{I}}-2 f(\mathbf{x})\|\mathbf{x}\|^{2}\left(2 \frac{\overline{\mathbf{x}}_{\mathcal{I}} \overline{\mathbf{x}}_{\mathcal{I}}^{\top}}{\|\mathbf{x}\|^{2}}+I_{\mathcal{I}}\right) \succeq O,
$$

which can be also written as

$$
X_{\mathcal{I}} A_{\mathcal{I I}} X_{\mathcal{I}} \succeq f(x)\|\mathbf{x}\|^{2}\left(2 \frac{\overline{\mathbf{x}}_{\mathcal{I}} \overline{\mathbf{x}}_{\mathcal{I}}^{\top}}{\|\mathbf{x}\|^{2}}+I_{\mathcal{I}}\right)-\frac{1}{2} \operatorname{Diag}\{A X \mathbf{x}\}_{\mathcal{I}} .
$$

This establishes the assertion.

\subsection{Quotient formulation vs. StQP: relations among solutions}

Theorem $\mathbf{5}$ A point $\overline{\mathbf{y}}$ is a local minimizer of problem (1) if and only if $\overline{\mathbf{x}}=T_{R}^{-1}(\overline{\mathbf{y}})$ is a local minimizer of problem (7). Further, a point $\overline{\mathbf{y}}$ is a global minimizer of problem (1) if and only if $\overline{\mathbf{x}}=T_{R}^{-1}(\overline{\mathbf{y}})$ is a global minimizer of problem (7). 
Proof. The transformation $\mathbf{y}=T_{R}(\mathbf{x})$ and its (partial) inverse $\mathbf{x}=T_{R}^{-1}(\mathbf{y})$ is well-defined and continuous. Moreover we have that $\mathbf{y}=T_{R}(\mathbf{x})$ is feasible for Problem (1) and $f(\mathbf{x})=\varphi\left(T_{R}(\mathbf{x})\right)$. Further $f\left(T_{R}^{-1}(\mathbf{y})\right)=\varphi(\mathbf{y})$, hence the result.

Theorem 6 Let $\overline{\mathbf{y}}$ be a KKT point of problem (1), then $\overline{\mathbf{x}}=T_{R}(\overline{\mathbf{y}})$ is a stationary point of problem (7).

Proof. Since $\overline{\mathbf{y}}$ is feasible, we have that $\|\overline{\mathbf{x}}\|^{2}=1$ and also that

$$
f(\overline{\mathbf{x}})=\frac{1}{2} \frac{\mathbf{x}^{\top} X A X \mathbf{x}}{\|\mathbf{x}\|_{2}^{4}}=\frac{1}{2} \overline{\mathbf{y}}^{\top} A \overline{\mathbf{y}}=\varphi(\overline{\mathbf{y}})
$$

The proof follows easily by observing that we can re-write equation (10) coordinate-wise as

$$
\overline{\mathbf{y}}_{i}\left[(A \overline{\mathbf{y}})_{i}-2 \varphi(\overline{\mathbf{y}})\right]=0,
$$

which is implied by (4) by identifying $\bar{\lambda}=-2 \varphi(\overline{\mathbf{y}})$.

The converse is not true, as the origin $\mathbf{o}$ is a stationary point. The loss of correspondence between KKT points implies that spurious stationary points can be created in passing from Problem (1) to Problem (7). However we can prove the following results.

Theorem $\mathbf{7}$ If $\overline{\mathbf{y}}$ is a KKT point for Problem (1) which satisfies the second-order necessary condition (5), then $\overline{\mathbf{x}}=T_{R}^{-1}(\overline{\mathbf{y}})$ is a stationary point for Problem (7), and the second-order necessary conditions SNR given in Theorem 4 are satisfied.

Proof. Let $\overline{\mathbf{y}}$ be a second order stationary point for Problem (1). Hence $\overline{\mathbf{x}}=\sqrt{\overline{\mathbf{y}}}$ is such that $\|\overline{\mathbf{x}}\|=1$ and by Theorem $6, \overline{\mathbf{x}}$ is a stationary point of Problem (7). Furthermore from (4), we get also that

$$
(A \bar{X} \overline{\mathbf{x}})_{i} \geq 2 f(\overline{\mathbf{x}}) \quad \text { for all } i \quad \text { such that } \quad \bar{x}_{i}=0 .
$$

Hence it remains to prove that

$$
\mathbf{d}_{\mathcal{I}}^{\top}\left(X_{\mathcal{I}} A_{\mathcal{I I}} X_{\mathcal{I}}-f(\mathbf{x})\left(2 \overline{\mathbf{x}}_{\mathcal{I}} \overline{\mathbf{x}}_{\mathcal{I}}^{\top}+I_{\mathcal{I}}\right)+\frac{1}{2} \operatorname{Diag}\{A X \mathbf{x}\}_{\mathcal{I}}\right) \mathbf{d}_{\mathcal{I}} \geq 0 \quad \text { for all } \mathbf{d} \in \mathbb{R}^{n} .
$$

First we observe that from the stationarity of $\overline{\mathbf{x}}$ we have that $(A X \mathbf{x})_{i}=2 f(\overline{\mathbf{x}})$ for all $i \in \mathcal{I}$, hence we can write the above condition as follows

$$
\mathbf{d}_{\mathcal{I}}^{\top}\left(X_{\mathcal{I}} A_{\mathcal{I I}} X_{\mathcal{I}}-2 f(\overline{\mathbf{x}}) \overline{\mathbf{x}} \overline{\mathbf{x}}^{\top}\right) \mathbf{d}_{\mathcal{I}} \geq 0 \quad \text { for all } \mathbf{d} \in \mathbb{R}^{n} .
$$

For any $\mathbf{d} \in \mathbb{R}^{n}$ we can define the vector $\mathbf{z}$ with components

$$
z_{i}= \begin{cases}0 & \text { if } \bar{x}_{i}=0, \\ \bar{x}_{i} d_{i}-\left(\overline{\mathbf{x}}^{\top} \mathbf{d}\right) \bar{x}_{i}^{2} & \text { if } \bar{x}_{i}>0 .\end{cases}
$$

which can be written as $\mathbf{z}=X(\mathbf{d}-\alpha \mathbf{x})$ with $\alpha=\overline{\mathbf{x}}^{\top} \mathbf{d}$. Hence, taking into account that $\|\overline{\mathbf{x}}\|^{2}=1$, we have

$$
\sum_{i \in \mathcal{I}(\overline{\mathbf{y}})} z_{i}=\sum_{i: \bar{x}_{i}>0} \bar{x}_{i} d_{i}-\left(\overline{\mathbf{x}}^{\top} \mathbf{d}\right) \sum_{i: \bar{x}_{i}>0} \overline{\mathbf{x}}_{i}^{2}=0
$$


where, as usual, $\mathcal{I}(\overline{\mathbf{y}})=\left\{i: \bar{y}_{i}>0\right\}$. Hence, by (6), we can write

$$
\mathbf{z}^{\top} A \mathbf{z}=(\mathbf{d}-\alpha \overline{\mathbf{x}})^{\top} \bar{X} A \bar{X}(\mathbf{d}-\alpha \overline{\mathbf{x}})=\mathbf{d}^{\top} \bar{X} A \bar{X} \mathbf{d}-2 \alpha \mathbf{d}^{\top} \bar{X} A \bar{X} \overline{\mathbf{x}}+\alpha^{2} \overline{\mathbf{x}}^{\top} \bar{X} A \bar{X} \overline{\mathbf{x}} \geq 0 .
$$

Recalling that $\bar{X} A \bar{X} \overline{\mathbf{x}}=2\|\overline{\mathbf{x}}\|^{2} f(\overline{\mathbf{x}}) \overline{\mathbf{x}}$ we can write

$$
\mathbf{d}^{\top} \bar{X} A \bar{X} \mathbf{d}-4 \alpha\|\overline{\mathbf{x}}\|^{2} f(\overline{\mathbf{x}}) \mathbf{d}^{\top} \overline{\mathbf{x}}+2 \alpha^{2}\|\overline{\mathbf{x}}\|^{2} f(\overline{\mathbf{x}}) \overline{\mathbf{x}}^{\top} \overline{\mathbf{x}} \geq 0
$$

recalling again $\|\overline{\mathbf{x}}\|=1$

$$
\mathbf{d}^{\top} \bar{X} A \bar{X} \mathbf{d}-2 f(\overline{\mathbf{x}})\left(\mathbf{d}^{\top} \overline{\mathbf{x}}\right)^{2}=\mathbf{d}^{\top}\left(\bar{X} A \bar{X}-2 f(\overline{\mathbf{x}}) \overline{\mathbf{x}} \overline{\mathbf{x}}^{\top}\right) \mathbf{d} \geq 0
$$

which is exactly the condition (11).

Theorem 8 If $\overline{\mathbf{x}} \neq \mathbf{o}$ is a stationary point for Problem (7), and the second-order necessary conditions SNR given in Theorem 4 are satisfied, then $\overline{\mathbf{y}}=T_{R}(\overline{\mathbf{x}})$ is a KKT point of Problem (1) which satisfies the second-order necessary condition (5).

Proof. The point $\overline{\mathbf{y}}=\bar{X} \overline{\mathbf{x}} /\|\overline{\mathbf{x}}\|^{2}$ is feasible for problem (1). By the first condition of Theorem 4 we get immediately that $\overline{\mathbf{y}}$ is a KKT point of Problem (1). Now assume that (11) holds for any d. Let $\mathbf{z}$ such that $z_{i}=0$ for $i: \bar{x}_{i}=0$ and $\mathbf{e}^{\top} \mathbf{z}=0$. Let us define a vector

$$
d_{i}= \begin{cases}0 & \text { if } \bar{x}_{i}=0 \\ \frac{z_{i}}{\bar{x}_{i}} & \text { if } \bar{x}_{i}>0\end{cases}
$$

Then we get $\bar{X} \mathbf{d}=\mathbf{z}$ and furthermore, since $d_{i}=0$ for all $i \notin \mathcal{I}(\overline{\mathbf{y}})$ by definition,

$$
\mathbf{d}^{\top} \overline{\mathbf{x}}^{\top}=\sum_{i: \bar{y}_{i}>0} d_{i} \overline{\mathbf{x}}_{i}=\sum_{i: \bar{x}_{i}>0} \overline{\mathbf{x}}_{i} \frac{z_{i}}{\overline{\mathbf{x}}_{i}}=\sum_{i \in \mathcal{I}(\overline{\mathbf{y}})} z_{i}=0 .
$$

Hence we can write

$$
\mathbf{d}^{\top}\left[\bar{X} A \bar{X}-2 f(\overline{\mathbf{x}}) \overline{\mathbf{x}} \overline{\mathbf{x}}^{\top}\right] \mathbf{d}=\mathbf{z}^{\top} A \mathbf{z}-2 f(\overline{\mathbf{x}}) \mathbf{d}^{\top} \overline{\mathbf{x}} \overline{\mathbf{x}}^{\top} \mathbf{d}=\mathbf{z}^{\top} A \mathbf{z} \geq 0,
$$

and the claim is proved.

\section{A quartic unconstrained formulation}

\subsection{An exact penalization method for StQP}

The unconstrained formulation of this section is based on results presented in [3]. In this paper, equivalence between StQP and a the minimization of a quadratic function over the positive orthant has been proved, which corresponds to an exact penalization of the linear constraint $\mathbf{e}^{\top} \mathbf{y}=1$. To be more precise, Problem (1) is equivalent to, the following quadratic problem over the positive orthant (OQP)

$$
\min \left\{h(\mathbf{p})=\frac{1}{2} \mathbf{p}^{\top} C \mathbf{p}-\mathbf{e}^{\top} \mathbf{p}: \mathbf{p} \geq \mathbf{o}\right\}
$$


where $C=\frac{1}{2} A+\gamma \mathbf{e} \mathbf{e}^{\top}$ and $\gamma$ is chosen such that $C$ is strictly $\mathbb{R}_{+}^{n}$-copositive.

In [3] has been proved the following result (note that the problem in [3] is in maximization form).

Theorem 9 (Correspondence among minimizers of StQP and (OQP)) Assume that $C$ is strictly $\mathbb{R}_{+}^{n}$-copositive and $C=\frac{1}{2} A+\gamma \mathbf{e e}^{\top}$. Then local and global solution of the $Q P$ (12) and of StQP (1) are related as follows.

(a) If $\overline{\mathbf{y}} \in \Delta$ is a local minimizer of Problem (1), then $\overline{\mathbf{p}}=\frac{1}{\gamma+\varphi(\overline{\mathbf{y}})} \overline{\mathbf{y}}$ is a local minimizer of Problem (12).

(b) If $\bar{p} \geq \mathbf{o}$ is a local minimizer of Problem (12), then $\overline{\mathbf{y}}=\frac{1}{\mathbf{e}^{\top} \overline{\mathbf{p}}} \overline{\mathbf{p}}$ is a local minimizer of Problem (1).

(c) The objective values in cases (a) and (b) are related by

$$
\frac{1}{\gamma+\varphi(\overline{\mathbf{y}})}=-2 h(\overline{\mathbf{p}}) \text {. }
$$

We complement this result by a study of correspondence of the KKT points.

\section{Theorem 10 (Correspondence among KKT points of StQP and (OQP))}

(a) If $\overline{\mathbf{y}} \in \Delta$ is a KKT point of Problem (1), then $\overline{\mathbf{p}}=\frac{1}{\gamma+\varphi(\overline{\mathbf{y}})} \overline{\mathbf{y}}$ is a KKT point of Problem (12).

(b) If $\overline{\mathbf{p}} \geq \mathbf{O}$ is a KKT point of Problem (12), then $\overline{\mathbf{y}}=\frac{1}{\mathbf{e}^{\top} \overline{\mathbf{p}}} \overline{\mathbf{p}}$ is a KKT point of Problem (1).

Proof. First let us write the KKT conditions for problem (12). A point $\overline{\mathbf{p}}$ is a KKT for problem (12) if $\overline{\boldsymbol{\mu}} \in \mathbb{R}^{n}$ exists such that

$$
\begin{aligned}
& C \overline{\mathbf{p}}-\mathbf{e}-\overline{\boldsymbol{\mu}}=\mathbf{o} \\
& \overline{\boldsymbol{\mu}}^{\top} \overline{\mathbf{p}}=0 \\
& \overline{\boldsymbol{\mu}} \geq \mathbf{o}, \quad \overline{\mathbf{p}} \geq \mathbf{o} .
\end{aligned}
$$

Hence we can also write

$$
\begin{aligned}
& \overline{\boldsymbol{\mu}}=C \overline{\mathbf{p}}-\mathbf{e} \geq \mathbf{o} \\
& \overline{\mathbf{p}}^{\top}(C \overline{\mathbf{p}}-\mathbf{e})=\overline{\mathbf{p}}^{\top} \overline{\boldsymbol{\mu}}=0 \\
& \overline{\mathbf{p}} \geq \mathbf{o} .
\end{aligned}
$$

Let us prove part (a). Let $\overline{\mathbf{y}} \in \Delta$ be a KKT point of Problem (1), and let $\overline{\mathbf{p}}=\frac{1}{\gamma+\varphi(\overline{\mathbf{y}})} \overline{\mathbf{y}}$. First we note that $\gamma+\varphi(\overline{\mathbf{y}})=\overline{\mathbf{y}}^{\top} C \overline{\mathbf{y}}>0$ due to strict copositivity of $C$. Hence $\bar{p}_{i} \geq 0$ iff $\overline{\mathbf{y}}_{i} \geq 0$. Now we calculate the coordinates of $C \overline{\mathbf{p}}-\mathbf{e}$ :

$$
\begin{aligned}
(C \overline{\mathbf{p}})_{i}-1= & \frac{1}{\gamma+\varphi(\overline{\mathbf{y}})}(C \overline{\mathbf{y}})_{i}-1=\frac{1}{\gamma+\varphi(\overline{\mathbf{y}})}\left[(C \overline{\mathbf{y}})_{i}-(\gamma+\varphi(\overline{\mathbf{y}}))\right] \\
& =\frac{1}{\gamma+\varphi(\overline{\mathbf{y}})}\left[\frac{1}{2}(A \overline{\mathbf{y}})_{i}+\gamma \mathbf{e}^{\top} \overline{\mathbf{y}}-\gamma-\varphi(\overline{\mathbf{y}})\right]=\frac{1}{2(\gamma+\varphi(\overline{\mathbf{y}}))}\left[(A \overline{\mathbf{y}})_{i}-2 \varphi(\overline{\mathbf{y}})\right] \\
& =\frac{1}{2(\gamma+\varphi(\overline{\mathbf{y}}))}\left[(A \overline{\mathbf{y}})_{i}+\bar{\lambda}\right] \geq 0
\end{aligned}
$$


where the last inequality derives from KKT for the simplex problem, recalling that $\bar{\lambda}=-2 \varphi(\overline{\mathbf{y}})$. Complementarity follows from the definition of $\overline{\mathbf{p}}$. Hence we get the first result. To prove part (b), let now $\overline{\mathbf{p}} \geq \mathbf{o}$ be a KKT point of problem (12) and define $\overline{\mathbf{y}}=\frac{1}{\mathrm{e}^{\top} \overline{\mathbf{p}}} \overline{\mathbf{p}}$. Of course $\overline{\mathbf{y}}$ is feasible for problem (1) and $\bar{y}_{i}>0 \Longleftrightarrow \bar{p}_{i}>0$ (note also that $\overline{\mathbf{p}} \neq \mathbf{o}$ ). Then we can write

$$
(A \overline{\mathbf{y}})_{i}+\bar{\lambda}=\frac{1}{\mathbf{e}^{\top} \overline{\mathbf{p}}}(A \overline{\mathbf{p}})_{i}-\overline{\mathbf{y}}^{\top} A \overline{\mathbf{y}}=\frac{1}{\mathbf{e}^{\top} \overline{\mathbf{p}}}(A \overline{\mathbf{p}})_{i}-\frac{1}{\left(\mathbf{e}^{\top} \overline{\mathbf{p}}\right)^{2}} \overline{\mathbf{p}}^{\top} A \overline{\mathbf{p}}=\frac{1}{\mathbf{e}^{\top} \overline{\mathbf{p}}}\left[(A \overline{\mathbf{p}})_{i}-\frac{1}{\left(\mathbf{e}^{\top} \overline{\mathbf{p}}\right)} \overline{\mathbf{p}}^{\top} A \overline{\mathbf{p}}\right] .
$$

¿From the KKT condition (13) we know that $\overline{\mathbf{p}}^{\top} C \overline{\mathbf{p}}-\overline{\mathbf{p}}^{\top} \mathbf{e}=0$ and substituting the expression of $C$ we get

$$
\overline{\mathbf{p}}^{\top} A \overline{\mathbf{p}}=-2\left[\gamma\left(\mathbf{e}^{\top} \overline{\mathbf{p}}\right)^{2}-\overline{\mathbf{p}}^{\top} \mathbf{e}\right]
$$

Then we can further write

$$
\begin{aligned}
(A \overline{\mathbf{y}})_{i}+\bar{\lambda} & =\frac{1}{\mathbf{e}^{\top} \overline{\mathbf{p}}}\left[(A \overline{\mathbf{p}})_{i}+\frac{2}{\left(\mathbf{e}^{\top} \overline{\mathbf{p}}\right)}\left(\gamma\left(\mathbf{e}^{\top} \overline{\mathbf{p}}\right)^{2}-\overline{\mathbf{p}}^{\top} \mathbf{e}\right)\right] \\
& \left.=\frac{2}{\mathbf{e}^{\top} \overline{\mathbf{p}}}\left[\frac{1}{2}(A \overline{\mathbf{p}})_{i}+\gamma\left(\mathbf{e}^{\top} \overline{\mathbf{p}}\right)-1\right)\right] \\
& \left.\left.=\frac{2}{\mathbf{e}^{\top} \overline{\mathbf{p}}}\left[\left(\frac{1}{2} A \overline{\mathbf{p}}+\gamma \mathbf{e} \mathbf{e}^{\top} \overline{\mathbf{p}}\right)_{i}-1\right)\right]=\frac{2}{\mathbf{e}^{\top} \overline{\mathbf{p}}}\left[(C \overline{\mathbf{p}})_{i}-1\right)\right] \geq 0 .
\end{aligned}
$$

Hence also the second part is proved.

Using formulation (12), we can define a new unconstrained formulation of the StQP.

By using the substitution $p_{i}=x_{i}^{2}$, to get rid of the sign constraints $p_{i} \geq 0$, we obtain the following unconstrained quartic formulation (QUP)

$$
\min \left\{\Psi(\mathbf{x})=\frac{1}{2} \mathbf{x}^{\top} X C X \mathbf{x}-\|\mathbf{x}\|^{2}: \mathbf{x} \in \mathbb{R}^{n}\right\} .
$$

Using the definition of $C$, we can write also

$$
\Psi(\mathbf{x})=\frac{1}{4} \mathbf{x}^{\top} X A X \mathbf{x}+\|\mathbf{x}\|^{2}\left(\frac{1}{2} \gamma\|\mathbf{x}\|^{2}-1\right)=\frac{1}{2} \Phi(\mathbf{x})+\frac{1}{2} \gamma\|\mathbf{x}\|^{4}-\|\mathbf{x}\|^{2} .
$$

Theorem 11 (Existence of unconstrained minimizer) Assume that $C$ is strictly $\mathbb{R}_{+}^{n}$-copositive and $C=\frac{1}{2} A+\gamma \mathbf{e e}^{\top}$. Then problem (14) admits a global minimizer.

Proof. We consider Problem (12) and consider the function $\psi_{\mathbf{y}}(t)=h(t \mathbf{y})$ along a ray emanating from 0 , where $\mathbf{y} \in \Delta$. Then $\psi_{\mathbf{y}}(t)=\frac{1}{2} t^{2} \mathbf{y}^{\top} C \mathbf{y}-t \mathbf{e}^{\top} \mathbf{y}$ is strictly convex and admits a minimizer at $t_{\mathbf{y}}=\frac{\mathbf{e}^{\top} \mathbf{y}}{\mathbf{y}^{\top} C \mathbf{y}}>0$, due to $\mathbf{y}^{\top} C \mathbf{y}>0$. The minimal value is $\psi_{\mathbf{y}}\left(t_{\mathbf{y}}\right)=-\frac{\left(\mathbf{e}^{\top} \mathbf{y}\right)^{2}}{2 \mathbf{y}^{\top} C \mathbf{y}}$, which is well defined and continuous in $\mathbf{y}$ over $\Delta$, so that

$$
\min \left\{\Psi(\mathbf{x}): \mathbf{x} \in \mathbb{R}^{n}\right\}=\min _{\mathbf{y} \in \Delta} \min \left\{\psi_{\mathbf{y}}(t): t \geq 0\right\}=-\max _{\mathbf{y} \in \Delta} \frac{\left(\mathbf{e}^{\top} \mathbf{y}\right)^{2}}{2 \mathbf{y}^{\top} C \mathbf{y}}
$$

is attained at some $\mathbf{y}^{*} \in \Delta$, so that a global solution to (14) is given by $x_{i}^{*}=\sqrt{t_{\mathbf{y}^{*}} y_{i}^{*}}$.

The quartic function $\Psi$ is twice continuously differentiable with gradient

$$
\nabla \Psi(\mathbf{x})=\frac{1}{2} \nabla \Phi(\mathbf{x})+2\left(\gamma\|\mathbf{x}\|^{2}-1\right) \mathbf{x}=X A X \mathbf{x}+2\left(\gamma\|\mathbf{x}\|^{2}-1\right) \mathbf{x}=2 X C X \mathbf{x}-2 \mathbf{x}
$$

and Hessian

$$
\begin{aligned}
\nabla^{2} \Psi(\mathbf{x}) & =\frac{1}{2} \nabla^{2} \Phi(\mathbf{x})+4 \gamma \mathbf{x} \mathbf{x}^{\top}+2\left(\gamma\|\mathbf{x}\|^{2}-1\right) I \\
& =4 X C X+2 \operatorname{Diag}\{C X \mathbf{x}\}-2 I=2 X A X+\operatorname{Diag}\{A X \mathbf{x}\}+4 \gamma \mathbf{x} \mathbf{x}^{\top}+2 \gamma\|\mathbf{x}\|^{2} I-2 I .
\end{aligned}
$$


Problem (14) is an unconstrained problem, hence we have the following well-known optimality conditions:

Theorem 12 (First and second order necessary condition for (QUP)) Let $\mathrm{x}$ be a unconstrained local minimizer of (14), then we have

$$
X A X \mathbf{x}+2\left(\gamma\|\mathbf{x}\|^{2}-1\right) \mathbf{x}=\mathbf{o}
$$

and

$$
2 X A X+\operatorname{Diag}\{A X \mathbf{x}\}+4 \gamma \mathbf{x} \mathbf{x}^{\top}+2\left(\gamma\|\mathbf{x}\|^{2}-1\right) I \succeq O
$$

\subsection{Quartic vs. StQPs: relationship among solutions}

Given any $\varepsilon \in \mathbb{R}$, we consider the sublevel set

$$
\mathcal{L}_{\varepsilon}=\left\{\mathbf{x} \in \mathbb{R}^{n}: \Psi(\mathbf{x}) \leq-\varepsilon\right\}
$$

Obviously, the origin $\mathbf{o} \in \mathcal{L}_{\varepsilon}$ for any $\varepsilon \leq 0$ but not for $\varepsilon>0$. Next, by strict $\mathbb{R}_{+}^{n}$-copositivity of $C=\frac{1}{2} A+\gamma \mathbf{e e}^{\top}$, there are constants $0<\delta<\rho$ such that

$$
\delta^{2} \leq \mathbf{y}^{\top} C \mathbf{y} \leq \rho^{2} \quad \text { for all } \mathbf{y} \in \mathbb{R}_{+}^{n} \text { with } \mathbf{e}^{\top} \mathbf{y}=1
$$

These constants can be estimated beforehand, and also somehow controlled by the choice of $\gamma$.

Now, for fixed $\mathbf{x} \in \mathbb{R}^{n}, s \geq 0$ and $\mathbf{y}=X \mathbf{x}$ we again consider $\psi_{\mathbf{y}}(s)=h(s \mathbf{y})=\Psi(\sqrt{s} \mathbf{x})=$ $\frac{s^{2}}{2} \mathbf{y}^{\top} C \mathbf{y}-s \mathbf{e}^{\top} \mathbf{y}$, which is a strictly convex quadratic function over $\mathbb{R}_{+}$with right-sided derivative

$$
\frac{\mathrm{d}}{\mathrm{d} s} \psi_{\mathbf{x}}(0+)=-\|\mathbf{x}\|^{2}<0 \quad \text { if } \quad \mathbf{x} \neq \mathbf{o} .
$$

This corroborates the fact that the origin $\mathbf{o}$ is rather a local maximizer of $\Psi$. Moreover, we can bound the sublevel set away from the origin, and at the same time establish compactness for (small enough) positive $\varepsilon$ :

Theorem 13 (Bounding sublevel sets) Let $0<\varepsilon<\frac{1}{2 \rho^{2}}$. Then

$$
\mathcal{L}_{\varepsilon} \subseteq\left\{\mathbf{x} \in \mathbb{R}^{n}: \delta^{-2}-\sqrt{\delta^{-4}-2 \varepsilon \delta^{-2}} \leq\|\mathbf{x}\|^{2} \leq \delta^{-2}+\sqrt{\delta^{-4}-2 \varepsilon \delta^{-2}}\right\} .
$$

Proof. Take any $\mathbf{x} \in \mathbb{R}^{n}$ with $\|\mathbf{x}\|^{2}=\mathbf{e}^{\top} \mathbf{y}=1$ and put $t=\left(\mathbf{y}^{\top} C \mathbf{y}\right)^{-1 / 2} \in\left[\frac{1}{\rho}, \frac{1}{\delta}\right]$, due to (17). Then it is easily checked that $\psi_{\mathbf{y}}(s) \leq-\varepsilon$ if and only if

$$
\phi_{-}(t) \leq s \leq \phi_{+}(t)
$$

where $\phi_{ \pm}(t)=t^{2} \pm \sqrt{t^{4}-2 \varepsilon t^{2}}$. Evidently, we have $\frac{\mathrm{d}}{\mathrm{d} t} \phi_{ \pm}(t)=2 t\left[1 \pm \frac{t^{2}-\varepsilon}{\sqrt{t^{4}-2 \varepsilon t^{2}}}\right]$, so that $\varepsilon<\frac{1}{2 \rho^{2}}$ implies that $\phi_{-}$is decreasing and $\phi_{+}$is increasing on the interval $\left[\frac{1}{\rho}, \frac{1}{\delta}\right]$. Therefore

$$
\phi_{-}\left(\frac{1}{\delta}\right) \leq \phi_{-}(t) \leq s=\|\sqrt{s} \mathbf{x}\|^{2} \leq \phi_{+}(t) \leq \phi_{+}\left(\frac{1}{\delta}\right) \text {, }
$$


which establishes the assertion.

Next let us define the transformations $\mathbf{y}=T_{Q}(\mathbf{x})$ with $y_{i}=\frac{1}{\|\mathbf{x}\|^{2}} x_{i}^{2}$ and $\mathbf{x}=\tilde{T}_{Q}(\mathbf{y})$ with $x_{i}=\sqrt{\frac{\left|y_{i}\right|}{\gamma+\varphi(\mathbf{y})}}$.

Theorem 14 Let $\mathbf{x}$ be a (local) global minimizer of problem (14) then $\mathbf{y}=T_{Q}(\mathbf{x})$ is a local (global) minimizer of problem (1). Viceversa, let $\mathbf{y}$ be a local (global) minimizer of problem (1) then $\mathbf{x}=\tilde{T}_{Q}(\mathbf{y})$ is a local (global) minimizer of problem (14).

Proof. Follows the same line of argumentation as for Theorem 5.

Theorem 15 Let $\mathbf{y}$ be a KKT point of problem (1) then $\mathbf{x}=\tilde{T}_{Q}(\mathbf{y})$ is a stationary point of problem (14).

Proof. The proof easily follows by observing that (15) can be written componentwise as

$$
x_{i}\left[(A X \mathbf{x})_{i}+2 \gamma\|\mathbf{x}\|^{2}-2\right]=0 .
$$

Substituting

we get

$$
x_{i}^{2}=\frac{\left|y_{i}\right|}{\gamma+\varphi(\mathbf{y})}=\frac{\left|y_{i}\right|}{\gamma-2 \bar{\lambda}}
$$

$$
\frac{1}{\gamma+\varphi(\mathbf{y})}\left[(A \mathbf{y})_{i}+\bar{\lambda}\right] x_{i}=0
$$

which is implied by (4).

The converse is not true. Actually the point $\overline{\mathbf{x}}=\mathbf{o}$ is stationary for Problem (14) but has obviously not a KKT counterpart for Problem (1). However, Theorem 13 tells us that the origin can be spared from our considerations, given we stay in a (reasonable) level set $\mathcal{L}_{\varepsilon}$. More generally speaking, the loss of correspondence between stationary/KKT points implies that spurious KKT points can be created in passing from Problem (1) to Problem (14). Fortunately, the reverse correspondence can be proved for refined KKT points of Problem (14) as follows.

Theorem 16 Let $\overline{\mathbf{x}}$ be a stationary point of problem (14) satisfying the second order necessary conditions. Then $\overline{\mathbf{x}} \neq \mathbf{o}$ and $\overline{\mathbf{y}}=\frac{1}{\|\overline{\mathbf{x}}\|^{2}} \bar{X} \overline{\mathbf{x}}$ is a KKT point of problem (1).

Proof. First note that, if (16) are satisfied, $\overline{\mathbf{x}} \neq \mathbf{o}$. Let $\bar{y}_{i}=\frac{1}{\|\overline{\mathbf{x}}\|^{2}} x_{i}^{2} \geq 0$ define a feasible point for Problem (1). We show that a $\bar{\lambda}$ exists such that (4) holds. First note that $\bar{y}_{i}=0$ if and only if $\bar{x}_{i}=0$. Next simply define $\bar{\lambda}=2\left(\gamma\|\overline{\mathbf{x}}\|^{2}-1\right)$. Then obviously $(A \overline{\mathbf{y}})_{i}=-\bar{\lambda}$ if $\bar{y}_{i}>0$. Further, if $\bar{y}_{i}=0$, then by the second-order condition, we get for the $i$ th diagonal element of the matrix in $(16)$

$$
(A \overline{\mathbf{y}})_{i}+\bar{\lambda}=(A \bar{X} \overline{\mathbf{x}})_{i}+2\left(\gamma\|\mathbf{x}\|^{2}-1\right) \geq 0
$$

which completes the proof. 


\section{Algorithmic aspects and numerical experience}

On the basis of the unconstrained formulations above, we have recast the problem of locating a constrained solution of the Standard Quadratic optimization problem (1) as the problem of locating an unconstrained solution of the function $P_{\varepsilon}$ from (3) or $f$ from (7) or $\Psi$ from (14). Note that the newly introduced unconstrained problems (7) and (14) have the advantage that they do not depend on a penalty parameter $\varepsilon$. Although in the quartic formulation (14) a parameter $\gamma$ appears in the definition of the matrix $C$, this is easily set knowing a range for $a_{i j}$.

For the solution of problems (7) or (14), we can use any unconstrained algorithm. Obviously standard unconstrained optimization methods produce stationary points and there is no guarantee that these correspond to global minimizers of the unconstrained function and hence of Problem (1). Then global optimization techniques must be adopted. We use a simple multi-start approach, repeating many local minimization processes starting from different, randomly chosen points and selecting the best obtained value. As for the unconstrained method, we use the non-monotone Barzilai-Borwein gradient method proposed in [9]. We coded the two alternative formulations in Fortran 90 and we test them on a set of benchmark problems arising from a continuous formulation of the classical maximum clique problem in graph theory. Given an undirected graph $G=(V, E)$ with vertex $V$ and edge set $E \subset V \times V$, the max clique problem consists on finding a complete subgraph of $G$ of maximum cardinality $\omega^{*}$. Among the different continuous formulation as a non convex optimization problem [5], we use the continuous formulation given by Bomze [2]. This is a regularization version of the Motzkin-Straus [13] formulation obtained by perturbing the objective function adding the term $\frac{1}{2}\|\mathbf{y}\|^{2}$, so that the maximum clique problem can be written as:

$$
\max \left\{\mathbf{y}^{\top}\left(A_{\mathcal{G}}+\frac{1}{2} I\right) \mathbf{y}: \mathbf{y} \in \Delta\right\}
$$

where $A_{\mathcal{G}}$ denotes the adjacency matrix of the graph, namely $a_{i j}=1$ if $(i, j) \in E$. The regularized version (18) avoids the drawback of the original Motzkin-Straus formulation of having spurious solutions, namely of solutions that are not in a one-to-one correspondence with solutions of the original combinatorial problems. Given a solution $\overline{\mathbf{y}}$ of (18), a vertex $i$ is in the clique if and only if $\bar{y}_{i}>0$ and the corresponding cardinality is $\bar{\omega}=\frac{1}{2}(1-\bar{f})^{-1}$. Obviously $\sigma^{*}$ is a maximum clique of $G$ is and only if $\mathbf{x}^{*}$ is the global solution of (18). In this case of problem (18) the choice of the parameter $\gamma$ in (14) is easily obtained noting that $-2 \leq a_{i j} \leq 0$ and $a_{i i}=-1$ (we have transformed the max problem into a min one), hence to obtained a strictly $\mathbb{R}_{+}^{n}$-copositive matrix with elements $c_{i j}=\frac{1}{2} a_{i j}+\gamma$, we can set $\gamma \geq 1$.

As a benchmark set, we use the 66 graph obtained from the DIMACS challenge [11]. Each problem has been solved starting with a randomly generated point $\mathbf{x}^{0}$ with $\left\|\mathbf{x}^{0}\right\|=1$. We perform 150 random runs. We compare the performance in terms of value of the clique found and computational time. In Table 1 we report the results in cumulative form. For each of the two formulations (7) or (14), we count the number of wins, ties and defeats over the 66 problems in terms of the largest clique size found, average clique size obtained, and cumulative computational time over 150 runs. Here, any difference smaller than $20 \%$ of the times between the formulations is considered as a tie. 


\begin{tabular}{|c|c|c|c|}
\hline & $\begin{array}{c}\text { best } \\
\text { results }\end{array}$ & $\begin{array}{c}\text { average } \\
\text { results }\end{array}$ & $\begin{array}{c}\text { less cpu time } \\
\text { over 150 runs }\end{array}$ \\
\hline winner (7) & 14 & 34 & 46 \\
\hline winner (14) & 7 & 27 & 17 \\
\hline tie & 45 & 5 & 3 \\
\hline
\end{tabular}

Table 1: Cumulative results of two different formulations for the 66 max-clique problems

On most problems, the two formulations are equivalent regarding the clique size found on the 150 runs (interestingly, this value was detected much earlier, typically after less than 20 runs). However, the quotient formulation (7) has better performance in terms of computational time. In Tables 2 and 3 we report the detailed results on the 66 problems in terms of the largest clique size found by using either the quotient formulation (7) or the quartic formulation (14). In the last column we report the largest known clique size or the clique number (if no $\geq$ sign is reported). Best results among the two formulations are shown in bold face.

\section{References}

[1] I.M. Bomze. Copositivity conditions for global optimality in indefinite quadratic programming problems. Czechoslovak Journal of Operations Research 1 7-19 (1992).

[2] I.M. Bomze. Evolution towards the maximum clique. Journal of Global Optimization 10 143-164 (1997).

[3] I.M. Bomze. On standard quadratic optimization problems. Journal of Global Optimization 13 369-387 (1998)

[4] I.M. Bomze. Quadratic optimization: standard problems; I - theory; II - algorithms; III - applications. In Encyclopedia of Optimization, 2nd ed., C.A. Floudas, P.M. Pardalos (eds.), Springer, New York, 2009.

[5] I.M. Bomze, M. Budinich, P. Pardalos, and M. Pelillo. The maximum clique problem. In Handbook of Combinatorial Optimization, D.-Z. Du, P.M. Pardalos (eds.), supp.Vol. A, pp. 1-74. Kluwer, New York, 1999.

[6] I. Bomze and L. Palagi. Quartic formulation of standard quadratic optimization. Journal of Global Optimization 32 181-205 (2005).

[7] S. Burer, R.D.C. Monteiro. A nonlinear programming algortihm for solving semidefinite programs via low-rank factorization. Mathematical Programming 95 329-357 (2003).

[8] R. Fletcher. Practical Methods of Optimization, Vol.2: Constrained Optimization. Wiley, New York, 1981.

[9] L. Grippo and M. Sciandrone. Nonmonotone globalization techniques for the Barzilai-Borwein gradient method. Computational Optimization and Applications 23 143-169 (2002).

[10] S. Homer and M. Peinado. Design and performance of parallel and distributed approximation algortihms for maxcut. Journal of Parallel and Distributed Computing 46 48-61 (1997). 
[11] D. Johnson and M.A. Trick, eds. Cliques Coloring and Satisfiability: Second DIMACS Implementation Challenge, vol. 26 of DIMACS Series, AMS 1996.

[12] J. Júdice, H. Sherali, and I. Ribeiro. The eigenvalue complementarity problem. Computational Optimization and Applications 37 139-156 (2007).

[13] T.S. Motzkin and E.G. Straus. Maxima for graphs and a new proof of a theorem of Turán. Canadian J. Math. 17 533-540 (1965). 


\begin{tabular}{|c|c|c|c|c|}
\hline graph & $n$ & $\begin{array}{c}\text { quotient } \\
\max \end{array}$ & $\begin{array}{c}\text { quartic } \\
\max \end{array}$ & $\begin{array}{c}\text { best } \\
\text { known }\end{array}$ \\
\hline brock200_2 & 200 & 10 & 10 & 12 \\
\hline brock200_3 & 200 & 13 & 13 & 15 \\
\hline brock200_4 & 200 & 15 & 15 & 17 \\
\hline brock400_1 & 400 & 24 & 22 & 27 \\
\hline brock400_2 & 400 & 22 & 22 & 29 \\
\hline brock400_3 & 400 & 21 & 22 & 31 \\
\hline brock400_4 & 400 & 23 & 23 & 33 \\
\hline brock800_1 & 800 & 18 & 18 & 23 \\
\hline brock800_2 & 800 & 18 & 18 & 24 \\
\hline brock800_3 & 800 & 17 & 17 & 25 \\
\hline brock800_4 & 800 & 18 & 18 & 26 \\
\hline c-fat $200-1$ & 200 & 12 & 12 & 12 \\
\hline c-fat $200-2$ & 200 & 24 & 24 & 24 \\
\hline c-fat $200-5$ & 200 & 58 & 58 & 58 \\
\hline c-fat $500-1$ & 500 & 14 & 14 & 14 \\
\hline c-fat500-10 & 500 & 126 & 126 & 126 \\
\hline c-fat $500-2$ & 500 & 26 & 26 & 26 \\
\hline c-fat $500-5$ & 500 & 64 & 64 & 64 \\
\hline hamming10-2 & 1024 & 370 & 363 & 512 \\
\hline hamming10-4 & 1024 & 33 & 32 & 40 \\
\hline hamming6-2 & 64 & 32 & 32 & 32 \\
\hline hamming6-4 & 64 & 4 & 4 & 4 \\
\hline hamming8-2 & 256 & 121 & 128 & 128 \\
\hline hamming8-4 & 256 & 16 & 16 & 16 \\
\hline johnson16-2-4 & 120 & 8 & 8 & 8 \\
\hline johnson32-2-4 & 496 & 16 & 16 & 16 \\
\hline johnson8-2-4 & 28 & 4 & 4 & 4 \\
\hline johnson8-4-4 & 70 & 14 & 14 & 14 \\
\hline keller4 & 171 & 11 & 9 & 11 \\
\hline keller5 & 776 & 19 & 19 & 27 \\
\hline keller6 & 3361 & 38 & 38 & $\geq 59$ \\
\hline
\end{tabular}

Table 2: DIMACS graphs: best results over 150 runs 


\begin{tabular}{|lc|c|c|c|}
\hline graph & $n$ & quotient & quartic & $\begin{array}{c}\text { best } \\
\text { max }\end{array}$ \\
known \\
\hline MANN_a27 & 378 & 122 & 122 & 126 \\
MANN_a45 & 1035 & 332 & $\mathbf{3 4 0}$ & 345 \\
MANN_a81 & 3321 & 1083 & $\mathbf{1 0 9 2}$ & 1100 \\
MANN_a9 & 45 & 16 & 16 & 16 \\
p_hat1000-1 & 1000 & 10 & 10 & $\geq 10$ \\
p_hat1000-2 & 1000 & 45 & 45 & $\geq 46$ \\
p_hat1000-3 & 1000 & 63 & 63 & $\geq 68$ \\
p_hat1500-1 & 1500 & 10 & 10 & $\geq 12$ \\
p_hat1500-2 & 1500 & $\mathbf{6 2}$ & 61 & $\geq 65$ \\
p_hat1500-3 & 1500 & 89 & 89 & $\geq 94$ \\
p_hat300-1 & 300 & 8 & 8 & 8 \\
p_hat300-2 & 300 & 25 & 25 & 25 \\
p_hat300-3 & 300 & 34 & 34 & 36 \\
p_hat500-1 & 500 & 9 & 9 & 9 \\
p_hat500-2 & 500 & $\mathbf{3 5}$ & 34 & 36 \\
p_hat500-3 & 500 & 48 & 48 & $\geq 50$ \\
p_hat700-1 & 700 & 9 & 9 & 11 \\
p_hat700-2 & 700 & 43 & 43 & $\geq 44$ \\
p_hat700-3 & 700 & 60 & $\mathbf{6 1}$ & $\geq 62$ \\
san1000 & 1000 & $\mathbf{9}$ & 8 & 15 \\
san200_0.7_1 & 200 & $\mathbf{1 7}$ & 16 & 30 \\
san200_0.7_2 & 200 & $\mathbf{1 4}$ & 13 & 18 \\
san200_0.9_1 & 200 & 48 & $\mathbf{5 1}$ & 70 \\
san200_0.9_2 & 200 & $\mathbf{4 7}$ & 44 & 60 \\
san200_0.9_3 & 200 & 34 & 34 & 44 \\
san400_0.5_1 & 400 & $\mathbf{8}$ & 7 & 13 \\
san400_0.7_1 & 400 & $\mathbf{2 2}$ & 21 & 40 \\
san400_0.7_2 & 400 & 17 & 17 & 30 \\
san400_0.7_3 & 400 & 15 & 15 & 22 \\
san400_0.9_1 & 400 & $\mathbf{5 6}$ & 55 & 100 \\
sanr200_0.7 & 200 & 17 & 17 & 18 \\
sanr200_0.9 & 200 & $\mathbf{3 9}$ & 38 & 42 \\
sanr400_0.5 & 400 & 12 & 12 & 13 \\
sanr400_0.7 & 400 & 18 & $\mathbf{1 9}$ & 22 \\
\hline
\end{tabular}

Table 3: DIMACS graphs: best results over 150 runs 